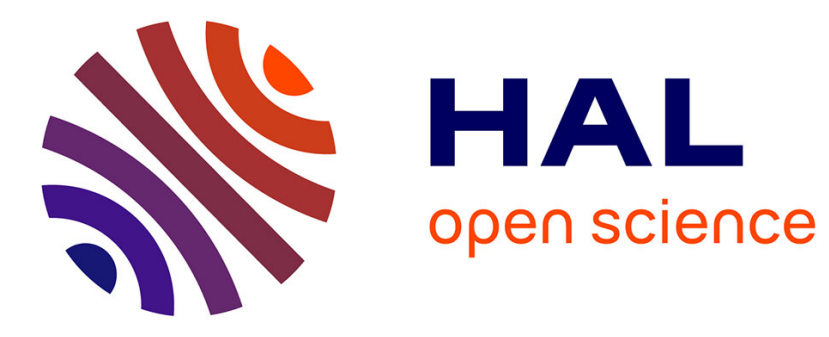

\title{
Self-Assembly of Bilayer Vesicles Made of Saturated Long Chain Fatty Acids
}

\author{
Jean-Paul Douliez, Bérénice Houinsou-Houssou, Anne-Laure Fameau, \\ Laurence Navailles, Frédéric Nallet, Axelle Grelard, Erick J. Dufourc, Cedric \\ Gaillard
}

\section{To cite this version:}

Jean-Paul Douliez, Bérénice Houinsou-Houssou, Anne-Laure Fameau, Laurence Navailles, Frédéric Nallet, et al.. Self-Assembly of Bilayer Vesicles Made of Saturated Long Chain Fatty Acids. Langmuir, 2016, 32 (2), pp. 401-410. 10.1021/acs.langmuir.5b03627 . hal-01368857

\section{HAL Id: hal-01368857 https://hal.science/hal-01368857}

Submitted on 29 Sep 2017

HAL is a multi-disciplinary open access archive for the deposit and dissemination of scientific research documents, whether they are published or not. The documents may come from teaching and research institutions in France or abroad, or from public or private research centers.
L'archive ouverte pluridisciplinaire HAL, est destinée au dépôt et à la diffusion de documents scientifiques de niveau recherche, publiés ou non, émanant des établissements d'enseignement et de recherche français ou étrangers, des laboratoires publics ou privés. 


\title{
Self-Assembly of Bilayer Vesicles Made of Saturated Long Chain Fatty Acids
}

\author{
Jean-Paul Douliez, ${ }^{* \dagger}{ }^{\dagger}$ Bérénice Houinsou Houssou, ${ }^{\ddagger}$ A-Laure Fameau, ${ }^{\ddagger}$ Laurence Navailles, ${ }^{\S}$ \\ Frédéric Nallet, ${ }^{\S}$ Axelle Grélard," Erick J. Dufourc," and Cédric Gaillard ${ }^{\ddagger}$ \\ ${ }^{\dagger}$ UMR 1332, biologie et pathologie du fruit, INRA, centre de Bordeaux, 33883 Villenave d'Ornon, France \\ ${ }^{\ddagger}$ UR 1268 Biopolymères Interactions et Assemblages, INRA, rue de la Géraudière, 44316 Nantes, France \\ ${ }^{\S}$ Centre de recherche Paul-Pascal, CNRS, and "Institute of Chemistry and Biology of Membranes and Nano-objects, UMR 5248, \\ CNRS, Université de Bordeaux, 33600 Pessac, France
}

Supporting Information

\begin{abstract}
Saturated long chain fatty acids (sLCFA, e.g., $\mathrm{C}_{14: 0}, \mathrm{C}_{16: 0}$, and $\left.\mathrm{C}_{18: 0}\right)$ are potentially the greenest and cheapest surfactants naturally available. However, because aqueous sodium soaps of sLCFA are known to crystallize, the self-assembly of stable bilayer vesicles has not been reported yet. Here, by using such soaps in combination with guanidine hydrochloride $(\mathrm{GuHCl})$, which has been shown recently to

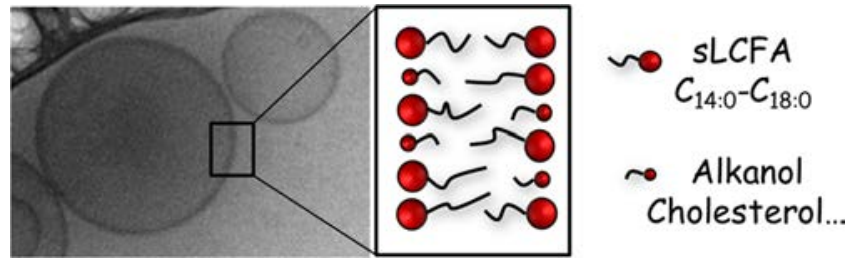
prevent crystallization, we were capable of producing stable bilayer vesicles made of sLCFA. The phase diagrams were established for a variety of systems showing that vesicles can form in a broad range of composition and $\mathrm{pH}$. Both solid state NMR and small-angle neutron scattering allowed demonstrating that in such vesicles sLCFA are arranged in a bilayer structure which exhibits similar dynamic and structural properties as those of phospholipid membranes. We expect these vesicles to be of interest as model systems of protocells and minimal cells but also for various applications since fatty acids are potentially substitutes to phospholipids, synthetic surfactants, and polymers.
\end{abstract}

\section{INTRODUCTION}

Vesicles find applications in various domains such as templates for biomineralization, ${ }^{1}$ drug delivery, and model of cells. ${ }^{2-5}$ They can form from aqueous dispersions of lipids, surfactants, and also polymers. Fatty acids are the simplest lipids and are of interest compared to synthetic surfactants because they are greener and bioavailable in large amounts and they naturally exist under various forms: having various chain lengths and possibly bearing double bonds and/or hydroxyl groups that may trigger the self-assembly. ${ }^{6}$ They are already known to exhibit good foaming and emulsifying properties ${ }^{6,7}$ that could be exploited for various applications. Unsaturated $\left(\mathrm{C}_{18: 1}\right)$ and short chain fatty acids (e.g., $<\mathrm{C}_{12: 0}$ ) have been shown to selfassemble into vesicles. ${ }^{8}$ Briefly, the above-mentioned fatty acids form micelles at high $\mathrm{pH}$ and addition of an alkanol or decreasing the $\mathrm{pH}$ yields the formation of vesicles. However, the formation of stable vesicles has not yet been demonstrated in the case of saturated long chain fatty acids (sLCFA, e.g., myristic acid $(\mathrm{Ma}): \mathrm{C}_{14: 0}$; palmitic acid $(\mathrm{Pa}): \mathrm{C}_{16: 0}$; and stearic acid (St): $\mathrm{C}_{18: 0}$ ). This is because sodium salts of sLCFA (soaps) have been known for a long time to crystallize below their Krafft point at room temperature. ${ }^{9-11}$ Then, vesicles that could eventually form by decreasing the $\mathrm{pH}$ or adding an alkanol are not stable and fatty acids crystallize. This is only 10 years ago that systems in which the sLCFA no longer crystallize have been developed. It requires the use of a "good counterion", for instance, tetrabutylammonium hydroxide $(\mathrm{TBAOH})$, instead of sodium or potassium as previously done, ${ }^{9-11}$ allowed dispersing in water sLCFA having chain length from $C_{14: 0}$ to $C_{24: 0}$. In these experimental conditions, sLCFA have been shown to form temperature-stable micelles; i.e., they could be dispersed in water without crystallizing. ${ }^{12,13}$ The Krafft point of these solutions was markedly decreased since micelles were still stable when samples were kept at $4{ }^{\circ} \mathrm{C}$ for long periods. Later, it was shown that choline, a greener counterion, could also yield stable micellar dispersions of sLCFA. ${ }^{14,15}$ Sodium salts of sLCFA have also been shown to form stable micelles and stacked membranes in water but upon addition of guanidine hydrochloride $(\mathrm{GuHCl}){ }^{16,17}$ The guanidium group is expected to strongly interact with the sLCFA carboxylate via electrostatic and bidendate interactions, ${ }^{6,18,19}$ thus preventing crystallization. We can then expect that if SLCFA can be dispersed in water by using a good counterion without crystallizing, stable vesicles could be obtained in such systems by varying experimental conditions. Here, we report such a finding using $\mathrm{GuHCl}$ and sLCFA-sodium soap dispersions. We do show that decreasing the $\mathrm{pH}$ or adding an alkanol allows forming stable vesicles. The term "stable" means here that vesicle dispersions can be kept for periods of month at $4{ }^{\circ} \mathrm{C}$ without observing any crystallization of sLCFA. Partial phase diagrams in various 
sLCFA systems show that vesicles can form in a broad range of composition and $\mathrm{pH}$. We also show by using solid state NMR and small-angle neutron scattering (SANS) that these vesicles are indeed formed by a fatty acid bilayer which exhibits properties similar as those observed for phospholipid membranes. Finally, critical vesicular concentrations (CVC) have been measured. We finally discuss our results and compare the sLCFA vesicles with those formed by using short or unsaturated fatty acid chains.

\section{MATERIALS AND METHODS}

Samples Preparation. Protonated (Sigma-Aldrich, Saint Quentin Fallavier, France) or deuterated (Eurisotop, Saclay, France) fatty acids, all of the highest purity, were weighted exactly in a tube, and ultrapure water was added depending on the desired concentration. Then, the desired volume of a $1 \mathrm{M}$ stock solution of guanidine hydrochloride (GuHCl, Sigma-Aldrich, Saint Quentin Fallavier, France, ref: G4505) and $\mathrm{NaOH}$ were added. In some cases, the commercial sodium salt (Sigma-Aldrich, Saint Quentin Fallavier, France) of fatty acids was used. In that case, only $\mathrm{GuHCl}$ was incorporated in the sample. All samples were submitted to at least three cycles of freeze thawing and further conserved at $-20^{\circ} \mathrm{C}$. Prior to be used, each sample was heated at $80^{\circ} \mathrm{C}$ for $10 \mathrm{~min}$. For samples in an excess of $\mathrm{GuHCl}$, the additional volume was poured, and sample tubes were again submitted to the above-mentioned procedure for homogenization. For all other samples including those with 1-decanol and 1-tetradecanol (all from SigmaAldrich, Saint Quentin Fallavier, France, and of the highest purity), the additional component was included, and samples were further again submitted to four cycles of freeze-thawing. In cholesterol (SigmaAldrich, Saint Quentin Fallavier, France) containing systems, samples were homogenized by 10 cycles of freeze thawing or by using sonication. In the latter case, a Vibra-Cell (Sonics and Materials Inc., Newtown, CT ) sonicator was used at power 5 in the continuous mode for $1 \mathrm{~min}$. Typically, five cycles of sonication were necessary, each separated by 5 min rest, while the sample was kept in an icewater bath.

Conductivity Measurements. Conductivity was measured at 25 ${ }^{\circ} \mathrm{C}$ using a conductometer from Atlantic labo-ics (France). Vesicle dispersions were produced as described above, and volumes of 10-100 $\mu \mathrm{L}$ were added step by step to a pure water initial solution. Conductivity was measured for each volume added after stabilization. The data were plotted as a function of the added volume and the concentration of sLCFA in solution. Determination of the CVC was done by manually drawing two straight lines. The concentration of sLCFA in Figure 5 is shown as [sLCFA], which stands for the total amount of lipids massed to prepare the vesicle solution; in other words, it includes both carboxylate and carboxylic forms of sLCFA.

Solid State NMR. For NMR experiments, perdeuterated sLCFA (Eurisotop, Gif-sur-Yvette, France) was used, and samples were prepared in the same way as previously mentioned except that only 1 $\mathrm{mL}$ was produced. Samples were placed into a $100 \mu \mathrm{L} \mathrm{ZrO}_{2}$ rotor (Cortecnet, Paris, France). Deuterium NMR experiments were carried out in the static mode at $76.8 \mathrm{MHz}$ for deuterium on a Bruker II Avance 500 WB spectrometer (Bruker, Wissembourg, France) with a CP-MAS dual $4 \mathrm{~mm}{ }^{1} \mathrm{H} /{ }^{2} \mathrm{H}$ probe. Quadrupolar-echo sequences ${ }^{20}$ were performed to record time-dependent signals that were Fourier transformed with $100-200 \mathrm{~Hz}$ Lorentzian filtering to yield wide-line spectra. Pulse durations and echo time delay were respectively 2.75 and $40 \mu \mathrm{s}$, the spectral window was $500 \mathrm{kHz}$, and $4 \mathrm{~K}$ acquisitions were accumulated with a repetition time of $2 \mathrm{~s}$.

Experimental (powder) NMR spectra were simulated using a Fortran routine (EJ Dufourc, unpublished): the time domain trace composed of the weighed sum of signals corresponding to each quadrupolar splitting of the entire lipid chain is calculated, and a Fourier transformation is performed, leading to simulated spectra. Adjustable parameters are the individual quadrupolar splittings and their intrinsic line width that can be measured at best on experimental spectra. All measurable quadrupolar splittings are taken as input parameters, the weight of each splitting being set according to the molecular structure (i.e., 1 for each of the $\mathrm{CD}$ group of the double bond, 2 for each $\mathrm{CD}_{2}$, and 3 for the terminal $\mathrm{CD}_{3}$ group). Comparison between experimental and calculated spectra is made until a satisfactory superimposition is obtained. ${ }^{21}$

Microscopy. Observations were made at room temperature at $20 \times$ magnification using an optical microscope in the phase contrast mode (Nikon Eclipse E-400, Tokyo, Japan) equipped with a 3-CCD JVC camera allowing digital images $(768 \times 512$ pixels $)$ to be collected. A drop of the lipid dispersion (about $20 \mu \mathrm{L}$ ) was deposited on the glass slide surface $(76 \times 26 \times 1.1 \mathrm{~mm}$, RS France $)$ and covered with a cover slide $(22 \times 22 \mathrm{~mm}$, Menzel-Glaser, Germany). The glass slides were previously cleaned with ethanol.

Cryo-TEM Experimental Procedure. All the cryo-TEM experiments were conducted as previously described. ${ }^{16}$ Briefly, specimens were prepared using a cryoplunge cryo-fixation device (Gatan, USA) on which a drop of the aqueous suspension was deposited on to glowdischarged holey-type carbon coated grid (Ted Pella Inc., USA). The grid was prepared by blotting the drop containing the specimen to a thin liquid layer remaining across the holes in the support carbon film. The liquid film was vitrified by rapidly plunging the grid into liquid ethane cooled with liquid nitrogen. The vitrified samples were mounted in a Gatan 910 specimen holder which was inserted in the microscope using a CT-3500-cryo transfer system (Gatan, USA) and cooled with liquid nitrogen. TEM images were then obtained from specimens preserved in vitreous ice and suspended across a hole in the supporting carbon substrate.

Small-Angle Neutron Scattering. Small-angle neutron scattering (SANS) experiments were performed at Laboratoire Léon-Brillouin (laboratoire mixte CEA/CNRS, Saclay, France) on spectrometer PAXY. The neutron beam was collimated by appropriately chosen neutron guides and circular apertures, with a beam diameter at the sample of $7.6 \mathrm{~mm}$. The neutron wavelength was set to 4 or $8 \AA$ with a mechanical velocity selector $(\Delta \lambda / \lambda \approx 0.1)$, the $2 \mathrm{D}$ detector $(128 \times$ 128 pixels, pixel size $5 \times 5 \mathrm{~mm}^{2}$ ) being positioned at 1.4 or $6.7 \mathrm{~m}$, respectively. The scattering wave vector, $Q$ then ranges from typically 0.006 to $0.5 \AA^{-1}$, with a significant overlap between the two configurations. The samples, prepared with deuterated water, were held in flat quartz cells with a $2 \mathrm{~mm}$ optical path and temperaturecontrolled by a circulating fluid to within $\pm 0.2{ }^{\circ} \mathrm{C}$. The azimuthally averaged spectra were corrected for solvent, cell, and incoherent scattering as well as for background noise. We thank Fabrice Cousin, our local contact, for his assistance during the neutron run.

\section{RESULTS}

Partial Phase Diagrams with Large Domains of Vesicles. As commented above, stable vesicles made of sLCFA have never been observed because sodium or potassium soaps were known to crystallize. We then used a system in which sLCFA no longer crystallizes. We started with stock solutions made of sLCFA, $\mathrm{NaOH}$, and $\mathrm{GuHCl}$ (further called sLCFA-NaGu) at an equimolar ratio $(1 / 1 / 1)$ at different sLCFA concentrations ranging from 0.5 to $300 \mathrm{mg} / \mathrm{mL}$. For all these stock solutions which had a $\mathrm{pH}$ of about 9.5, the dispersions were slightly turbid and made of bilayer sheets at low temperature and transited to isotropic viscous elongated micellar solutions at a temperature that depends on the alkyl chain length as previously commented. ${ }^{16,17}$ Systems composed by myristic acid ( $\left.\mathrm{My}, \mathrm{C}_{14: 0}\right)$ were mainly studied, but vesicles were also observed for systems made of palmitic $\left(\mathrm{Pa}, \mathrm{C}_{16: 0}\right)$ and stearic acid $\left(\mathrm{St}, \mathrm{C}_{18: 0}\right)$. Partial phase diagrams were established by visual inspection of the samples and phase contrast microscopy observations. We focused on domains for which only stable vesicles were observed. Vesicles are observed as circles and lamellar phases as sheets of membranes by phase contrast microscopy. When crystals form at too low $\mathrm{pH}$, they were easily visible by visual inspection of the sample tubes since 

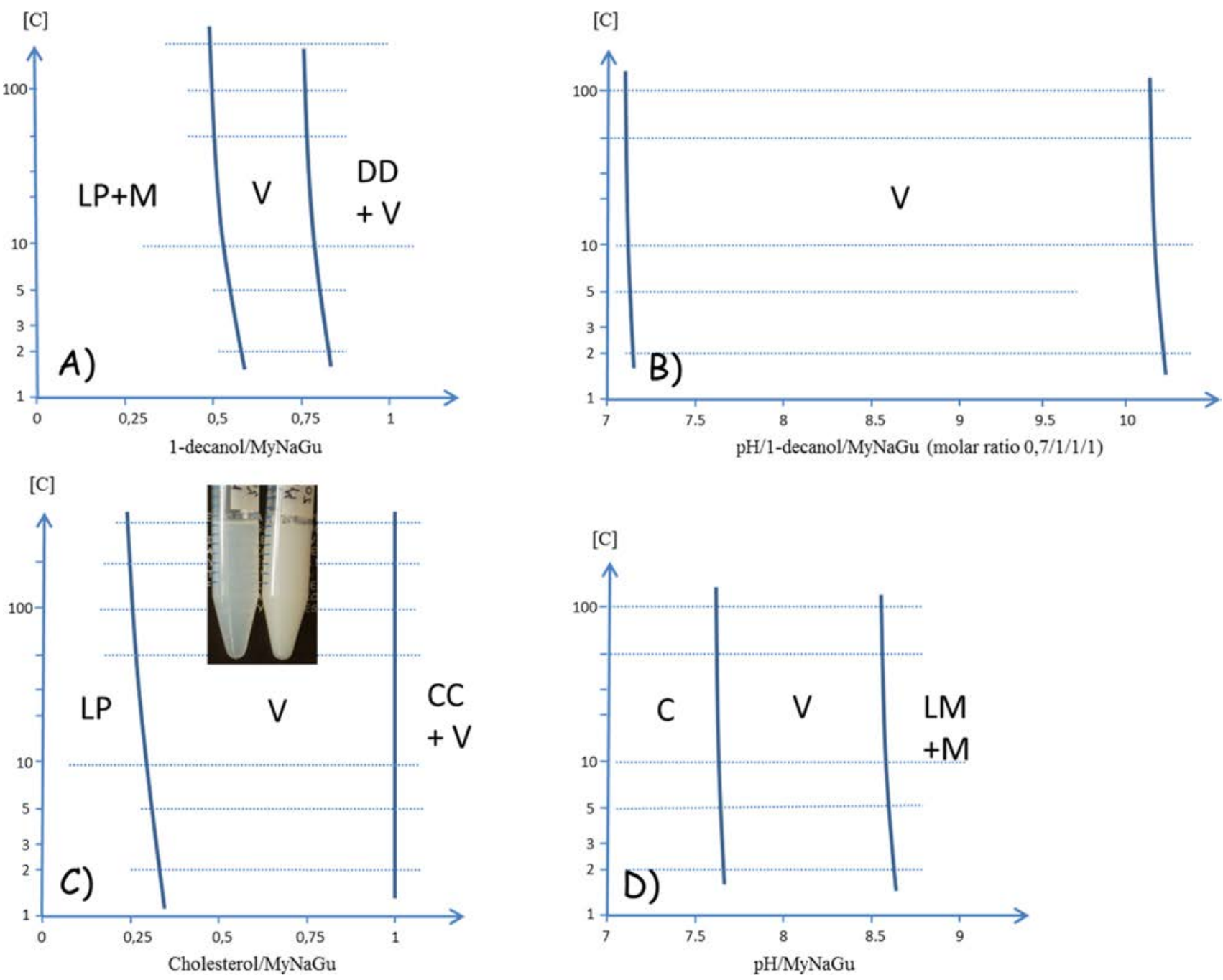

Figure 1. Partial phase diagrams in various sLCFA systems. The vesicle domain is indicated by a "V". (A) In the system MyNaGu and 1-decanol as a function of the molar ratio and the My concentration (LP: lamellar phase; M: possible presence of micelles; DD: presence of 1-decanol droplets). (B) In the system $\mathrm{MyNaGu}$ and 1-decanol as a function of $\mathrm{pH}$ and the My concentration. (C) In the system MyNaGu and cholesterol as a function of the molar ratio and the concentration of the My concentration (LP: lamellar phase; CC presence of cholesterol crystals). The inset shows a photograph of sample tubes prepared via the freeze-thawing method (right) and via sonication (left). (D) In the system MyNaGu as a function of $\mathrm{pH}$ and the My concentration (C: My crystals; LP and M as indicated above).

sediments were observed but also by phase contrast microscopy. All samples containing vesicles were placed at 4 ${ }^{\circ} \mathrm{C}$ for periods of months and further examined by phase contrast microscopy to confirm that vesicles were stable; i.e., no crystals were observed.

It is known in the case of unsaturated and short chain fatty acids that vesicles can form upon decreasing the $\mathrm{pH}$ of micellar solutions or upon addition of alkanols. ${ }^{22-24}$ sLCFA vesicles were then successfully, at first, produced upon addition of alkanol to sLCFA-NaGu stock solutions. Mixtures of 1-decanol and $\mathrm{MyNaGu}$ were prepared yielding the phase diagram represented Figure 1A. Because this alkanol is liquid but insoluble in water, vigorous shaking and freeze-thawing cycles were necessary to get homogeneous samples. Vesicles were stable for several months at $4{ }^{\circ} \mathrm{C}$ without evidence of the formation of any crystals. The phase diagram showed that vesicles were obtained for a relatively large domain of molar ratio between the alkanol and the sLCFA. For too low amounts of 1-decanol, mixtures yielded lamellar phases probably in coexistence with micelles although this can be confirmed neither by visual inspection nor by microscopy. However, since we focus on the domains in which only vesicles form, this part of the phase diagram was not further investigated in details. For an excess of alkanol, it could no longer be mixed with sLCFA and was thus found in coexistence with vesicles as free droplets (not shown).

The effect of $\mathrm{pH}$ in the system 1 -decanol/MyNaGu (molar ratio $0.7 / 1 / 1 / 1$ ) was also studied and is depicted Figure $1 \mathrm{~B}$. The $\mathrm{pH}$ of the initial vesicle solution containing 1-decanol was the same as that of the stock solution, i.e., 9.5 (see above). The $\mathrm{pH}$ was then first increased with $\mathrm{NaOH}(1 \mathrm{M})$, and samples were heated at $80{ }^{\circ} \mathrm{C}$ and vigorously shaken for homogenization. Vesicles were still observed for a $\mathrm{pH}$ of about 10.3, slightly depending on the fatty acid concentration. Because stock solutions are isotropic (above the transition, see above) and exhibit a $\mathrm{pH}$ of about 9.5, this means that 1-decanol stabilizes the vesicles at higher $\mathrm{pH}$. Above that value of 10.3, transparent isotropic solutions were obtained, suggesting that micelles formed at higher $\mathrm{pH}$ even in the presence of the alkanol. 

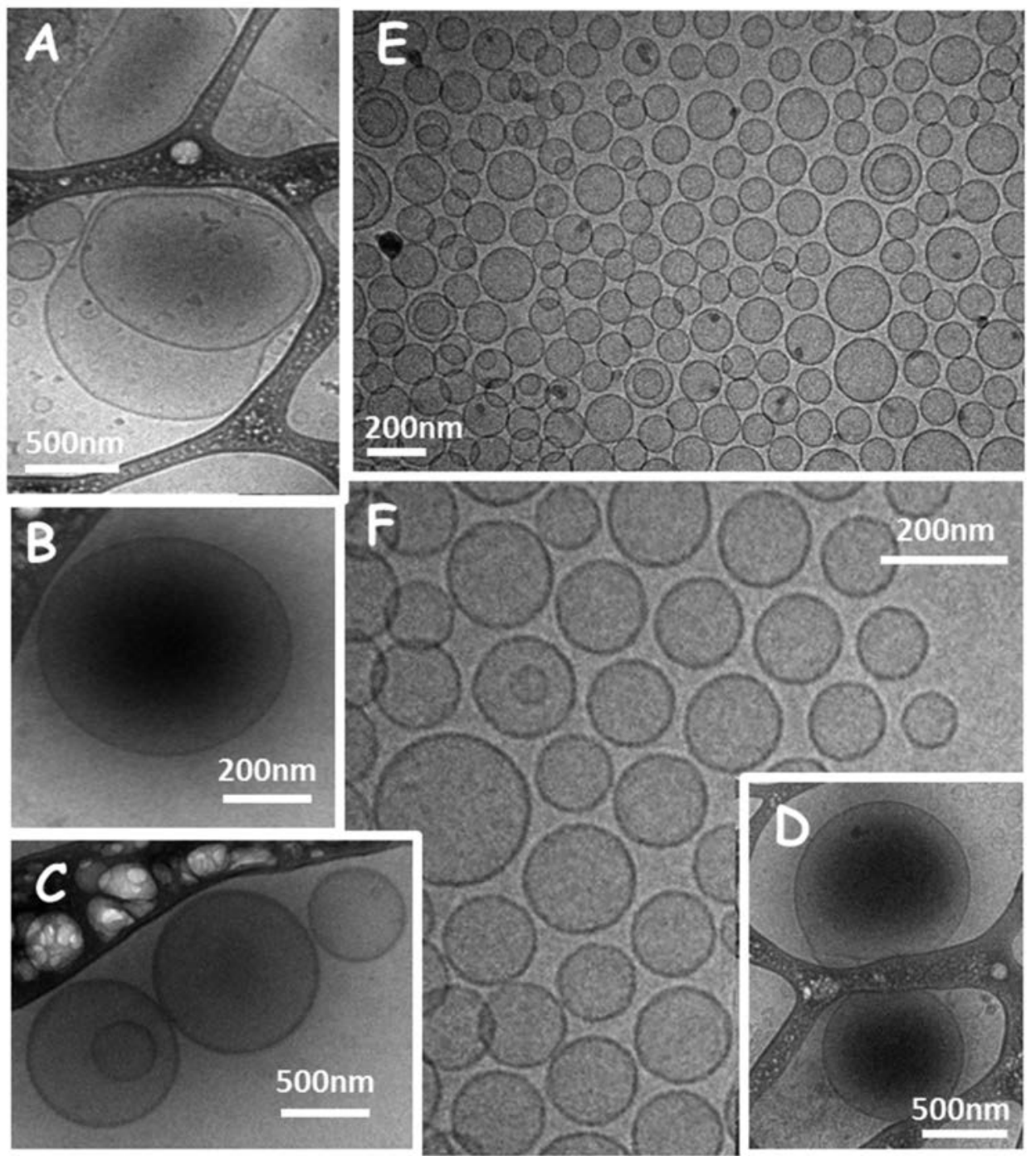

Figure 2. Cryo-TEM images of selected samples in various sLCFA systems. (A) In the system 1 -decanol/MyNaGu $(\mathrm{molar}$ ratio $0.7 / 1 / 1 / 1)$ at $\mathrm{pH}$ 9.5. (B) In the same system as above but at $\mathrm{pH}$ 8. (C) In the system MyNaGu at $\mathrm{pH}$ 8. (D) In the system PaNaGu at $\mathrm{pH}$ 8. (E) In the system $\mathrm{MyNaGu} /$ cholesterol (1/1/1/1) after sonication. (F) In the system MyNaGu in a Tris-buffer at $\mathrm{pH} 8$ after sonication.

Decreasing the $\mathrm{pH}$ was also investigated, and vesicles were still observed for a value of about 7.2, a value slightly lower than that in the absence of 1-decanol (see below). One also studied the systems sLCFA-NaGu mixed with cholesterol, the phase diagram of which is represented Figure $1 \mathrm{C}$ in the case of myristic acid. Generally, in phospholipid-containing cholesterol systems, both lipids are first dissolved in a solvent which is further dried under a stream of nitrogen. Hydration further yields homogeneous samples in the bilayer of which the sterol is well incorporated between phospholipid molecules. Here, a greener method was proposed since no solvents were used; the given amount of cholesterol was simply massed in a sample tube containing the sLCFA-NaGu dispersion. Vigorous shaking for $5 \mathrm{~min}$ and five cycles of freeze-thawing $(-20$ ${ }^{\circ} \mathrm{C} / 80{ }^{\circ} \mathrm{C}$ ) were only necessary to get well-dispersed systems. An alternative to the above-mentioned mixing procedure was to use ultrasonic power to fully disperse cholesterol (see Methods section). Samples produced by the sonication procedure were much less turbid (Figure 1C, inset). However, only one cycle of freezing-unfreezing $\left(-20{ }^{\circ} \mathrm{C} / 20{ }^{\circ} \mathrm{C}\right)$ yielded the same sample turbidity as that observed for samples prepared without sonication. Small size vesicles (see below) were then obtained upon sonication; they could be destabilized upon freezing, fusing to larger ones. Upon increasing the amount of sterol below a molar ratio $\mathrm{My} / \mathrm{chol}$ of 1 , vesicles were found to coexist with crystals that were attributed to cholesterol which could no longer be incorporated within the fatty acid bilayers.

As in the case of unsaturated and short chain fatty acids, sLCFA vesicles were also successfully produced by decreasing the $\mathrm{pH}$ of the initial stock solutions (Figure 1D). Addition of small volumes of concentrated $(1 \mathrm{M}) \mathrm{HCl}$ solution immediately yielded sLCFA precipitation, but freeze-thawing cycles allowed obtaining vesicles, again stable for months without appearance of any crystals. Below a $\mathrm{pH}$ of ca. 7.6, vesicles were still produced at room temperature, but they further crystallized upon resting at $4{ }^{\circ} \mathrm{C}$ (Figure 1D). Stable vesicles were also produced in a tris buffer $100 \mathrm{mM}, \mathrm{pH} 8.5$; in such a case, $\mathrm{NaOH}$ was not included, but $\mathrm{GuHCl}$ was still necessary to prevent the crystallization.

Two additional phase diagrams are shown in Supporting Information (S.I.1) displaying the domain of vesicle formation in mixtures of $\mathrm{MyNaGu}$ plus the monoglyceride derivative of 
a)

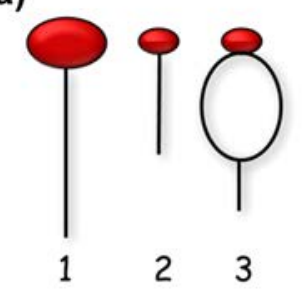

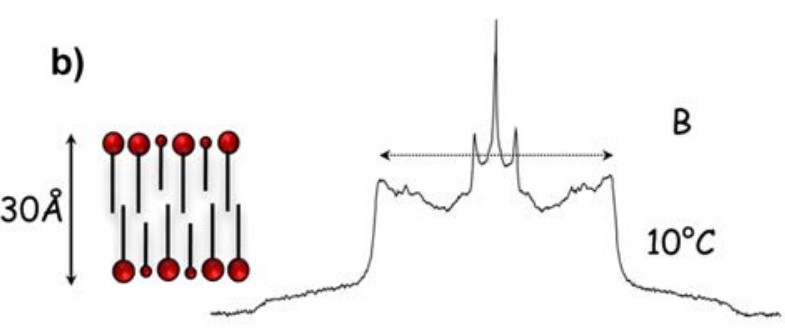

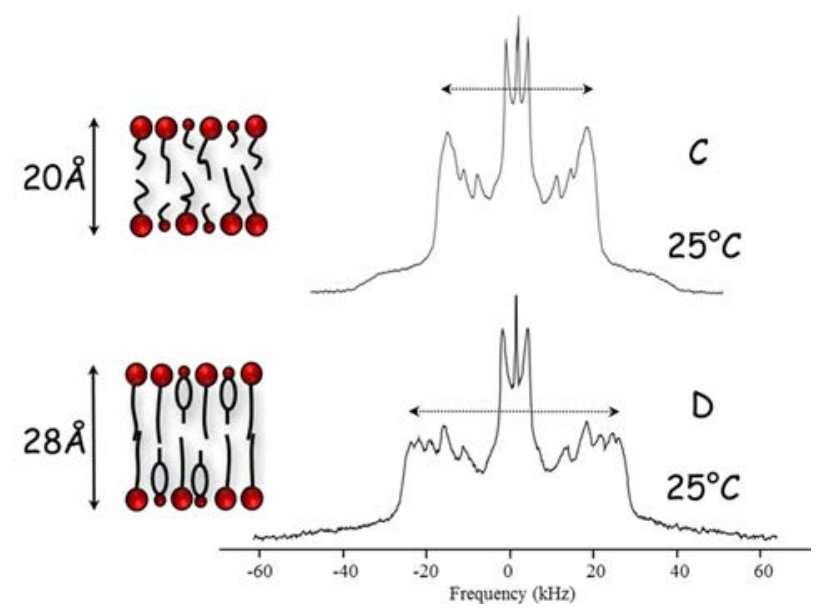

Figure 3. Physicochemical characterization of the vesicles. (A) Schematic representation of the compounds: $1=$ My, $2=1$-decanol, $3=$ cholesterol. (B) Solid state NMR spectra for selected systems, temperature being indicated on the right. The double arrow indicates the largest quadrupolar splitting. On the left is shown a schematic representation of the bilayer and the thickness as measured from the data is indicated (see text for details): $(1,2)$ in the system 1 -decanol/MyNaGu (molar ratio $0.7 / 1 / 1 / 1)$ at $\mathrm{pH} 9.5 ;(3)$ in the system cholesterol/MyNaGu $(\mathrm{molar}$ ratio $1 / 1 / 1 / 1)$ at $\mathrm{pH}$ 9.5.

My, namely, 1-tetradecanoylglycerol and also in mixtures of 1tetradecanol and StNaGu. It is noteworthy that for the latter system vesicles were observed for molar ratios alkanol/sLCFA much lower than in the case of the system 1-decanol/MyNaGu (see Figure 1A).

Microscopic Observations of Vesicles. Although the phase diagrams were established by using optical microscopy, one also performed cryo-TEM experiments using various vesicular solutions to get additional and more powerful information on their shape, multilamellarity, etc. (Figure 2). A general observation of images allowed demonstrating that vesicles indeed formed under various conditions. Polydisperse circles with a black contour attested the formation of vesicles with a dense electron diffusing matter (associated with the presence of sLCFA) delimiting the surface of volumes encapsulating water. Images showed that vesicles were mostly unilamellar, although some of them were also multilamellar. Sonication of these sLCFA dispersions yielded the formation of smaller size vesicles as observed in the case of phospholipid vesicles. It is noteworthy that although cryo-TEM is a powerful technique for confirming the presence of vesicles, the vitrified sample thickness which is about or less than $1 \mu \mathrm{m}$ excludes the presence of larger objects. Besides, larger vesicles of about 10 $\mu \mathrm{m}$ were clearly observed by phase contrast microscopy (not shown).

NMR and SANS Studies of the SLCFA Membranes Forming Vesicles. One then aimed at studying the sLCFA membrane of vesicles, the various components of which are schematized in Figure 3A. One first investigated vesicles made of $\mathrm{MyNaGu}(20 \mathrm{mg} / \mathrm{mL}$ of perdeuterated $\mathrm{My})$ and 1-decanol at a molar ratio $1 / 1 / 1 / 0.7$ (see above) by using deuterium solid state NMR. This technique is already well-known to be powerful for studying lipid membrane properties. $^{20}$ The spectrum for such vesicles recorded at $10{ }^{\circ} \mathrm{C}$ is shown Figure $3 \mathrm{~B}$. An isotropic line of weak intensity was observed as the result of the natural abundance deuterated water present in the sample since one did not use deuterated-depleted water. The spectrum showed a so-called axially symmetric "powder pattern" shape that is generally obtained for lipids embedded in bilayers. ${ }^{20}$ It resulted from the superimposition of the various signals arising from each $\mathrm{CD}_{2}$ groups along the acyl chain, here perdeuterated $\mathrm{My}$. The most intense peaks represent molecules, within the vesicles, oriented at $90^{\circ}$ with respect to the magnetic field and low-intensity shoulders to molecules oriented at $0^{\circ}$. Quadrupolar splittings at $90^{\circ}$ could be measured from this spectrum; the largest is highlighted in Figure $3 \mathrm{~B}$ by a double arrow that shows a value of about $58.5 \mathrm{kHz}$. This value is slightly lower than half of the static value that would be obtained in the absence of motions $(125 \mathrm{kHz})$, indicating that at such a temperature the acyl chains of myristic acid show limited dynamics. Smaller quadrupolar splittings could also be observed on the spectrum and could be assigned to $\mathrm{CD}_{2}$ groups at the end of the chain with the smallest being assigned to the rapidly rotating methyl terminal group. This suggested that the end of myristic acid chain exhibited some gauche defects. The particular feature of this spectrum is that it exhibits an axially symmetric pattern but also large quadrupolar splittings. It is noteworthy that in phospholipid systems bilayers in the $\mathrm{L}_{\beta}$ phase return NMR spectra with large quadrupolar splittings but unsymmetrical powder patterns. ${ }^{20}$ It is assumed in such a phase that the lipid chains are in an all-trans conformation and that the whole lipid does not exhibit axially rotation along its long axis. In phospholipid systems, NMR spectra exhibiting axially symmetric pattern and also large quadrupolar splittings are 

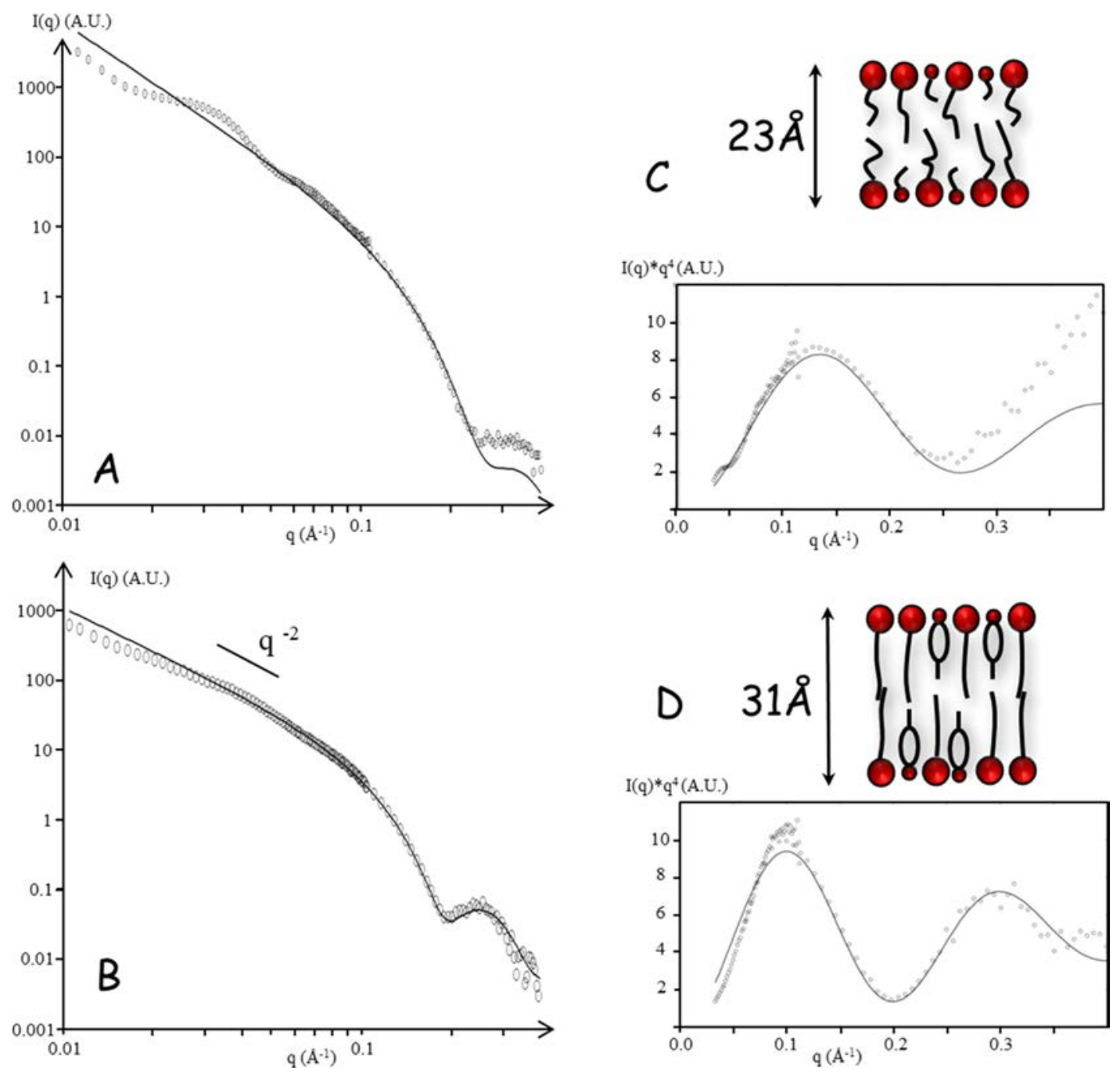

Figure 4. SANS spectra in the double-logarithmic $\left(I(q)\right.$ vs $q$ : A and B) and in the Porod representations $\left(I(q) q^{4}\right.$ vs $q$ : C and D) for the system 1decanol/MyNaGu (molar ratio 0.7/1/1/1) at pH 9.5 (A and C) and cholesterol/MyNaGu (molar ratio $1 / 1 / 1 / 1)$ at $\mathrm{pH} 9.5$ (B and D). The bilayer is schematically represented (see Figure $3 \mathrm{~A}$ for details), and the thickness as determined from the fit is also indicated (see text for details).

found in a particular phase called the "liquid ordered phase", $\mathrm{L}_{\mathrm{O}}$. This phase is generally found in systems that combines lipids and cholesterol and has been related to "lipid rafts". In such a phase, it is assumed that lipid chains are almost in an alltrans conformation, but the whole lipid does exhibit rotation along its long axis.

Here in the present sLCFA vesicles, one cannot conclude that decanol behave in a similar way as cholesterol does in phospholipid systems. Besides, NMR spectra exhibiting axially symmetric pattern and also large quadrupolar splittings are found in many other related pure sLCFA systems. ${ }^{6}$ Then, this seems to be a general feature in fatty acid systems. We believe that compared to phospholipids that bear two alkyl chains, fatty acids have only a single chain which can axially rotating along the long axis even at low temperature. We then suggest that in the present vesicles made of sLCFA and 1-decanol sLCFA are embedded in a $\mathrm{L}_{\beta}$ phase, although the spectrum was found symmetrical, suggesting the presence of axial rotational movements of fatty acids chain with respect to the bilayer normal.

For the same system but at $25{ }^{\circ} \mathrm{C}$, the spectrum exhibited a similar shape but with a markedly smaller width (Figure 3C). The largest quadrupolar splitting (double arrow) had a value of about $34.5 \mathrm{kHz}$, then much lower than that observed at $10{ }^{\circ} \mathrm{C}$, and several inner quadrupolar splittings could be detected and assigned to $\mathrm{CD}_{2}$ chain segments near the chain end. This suggests that at such a temperature myristic acids were embedded in a fluid $\mathrm{L}_{\alpha}$ phase. ${ }^{20}$ In such a phase, the lipid chains are known to exhibit gauche defects and are disordered, and the whole lipid also exhibits rotation along its long axis. The exact phase transition temperature that can be generally measured by using differential scanning calorimetry was not determined in our cases.

In the case of vesicles containing cholesterol, the spectrum (see Figure 3D) showed an "intermediate" width compared to that observed previously in its absence at $25{ }^{\circ} \mathrm{C}$. Compared to the above spectrum (Figure $3 \mathrm{C}$ ) at the same temperature, the width was rather large with the largest quadrupolar splitting of $54.5 \mathrm{kHz}$ (double arrow), and several peaks having smaller quadrupolar splittings could also be assigned. This feature was very similar to that observed for phospholipid systems containing cholesterol and suggested that myristic chains were embedded in a liquid ordered phase, $\mathrm{L}_{\mathrm{O}}$, as had already been shown for similar systems. ${ }^{25,26}$

All spectra were simulated using a homemade software (Dufourc and Douliez, unpublished) that returned the shape as 

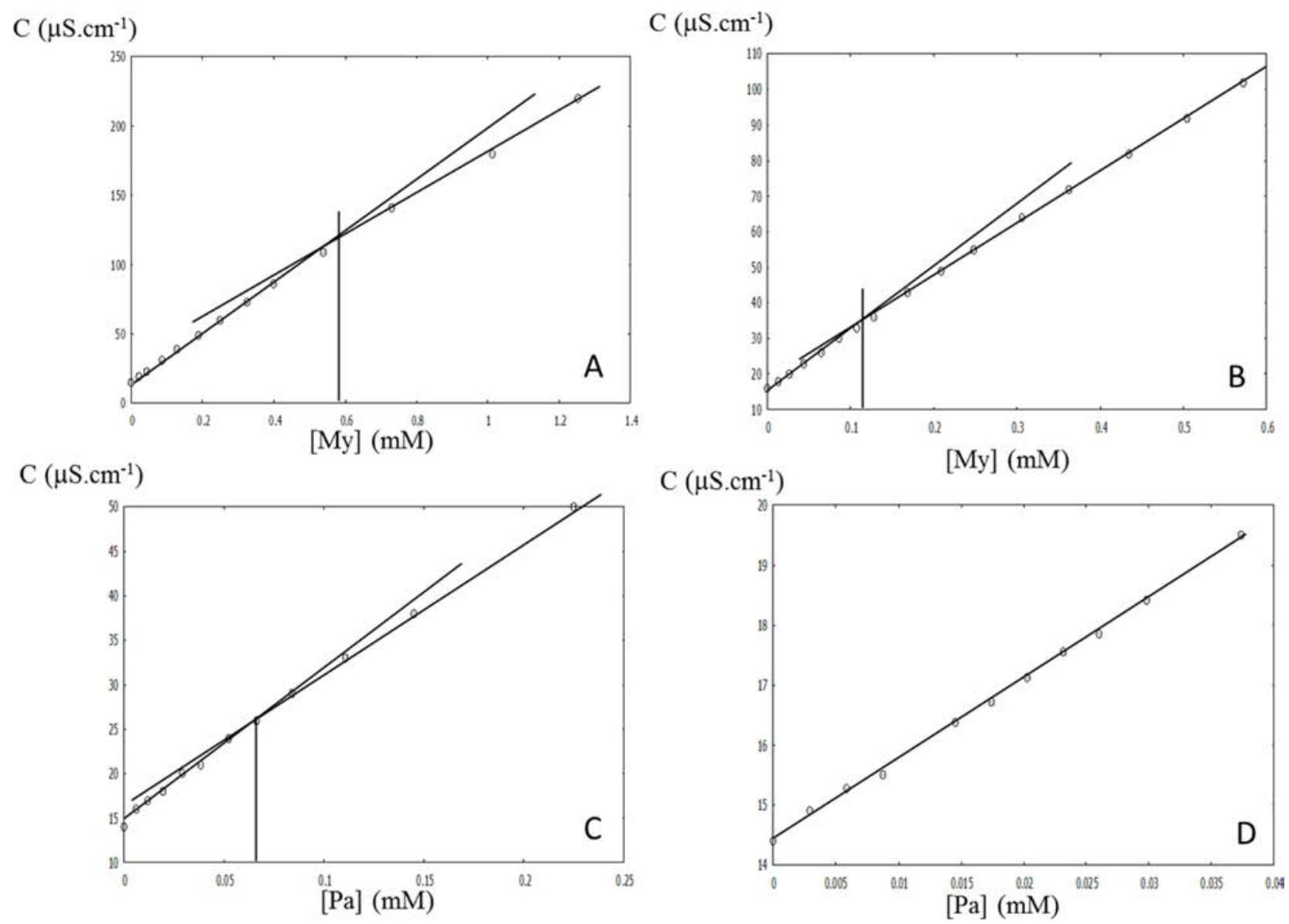

Figure 5. Variation of the conductivity, $C$ (in $\mu \mathrm{S} / \mathrm{cm}$ ), as a function of the sLCFA concentration in the system $\mathrm{MyNaGu}$ at $\mathrm{pH} 8$ (A), 1-decanol/ MyNaGu (molar ratio 0.7/1/1/1) at $\mathrm{pH} 8$ (B), 1-decanol/PaNaGu (molar ratio 0.7/1/1/1) at $\mathrm{pH} 8$ (C), and 1-dodecanol/PaNaGu (molar ratio $0.7 / 1 / 1 / 1)$ at $\mathrm{pH} 8(\mathrm{D})$.

a function of a set of quadrupolar splittings to be varied. The data were arbitrarily modified until a satisfactory superimposition was obtained between experimental and calculated spectra. The results are shown in the Supporting Information (S.I. 2) for the three above-mentioned systems. In all cases, a good agreement was obtained allowing drawing an order profile that returned the quadrupolar splitting vs the labeled carbon position (see S.I.2). It must be noticed that the assignment was done according to phospholipid systems ${ }^{20}$ and theoretical expectation ${ }^{27}$ which suggests a monotonic decrease of the quadrupolar splitting when going toward the methyl end. From these data, one can calculate the hydrophobic thickness of the bilayer with a simple geometrical model which allows measuring the acyl chain length as a function of the quadrupolar splittings. ${ }^{28}$ In this model, the hydrophobic thickness of one lipid layer is calculated from the quadrupolar splittings, and it is assumed that the hydrophobic thickness of the bilayer is twice (tail-to-tail conformation, no chain interdigitation). The data obtained are gathered on the schemes in Figure 3. For the system containing 1-decanol, the values were 30 and $20 \AA$ for vesicle bilayers at 10 and $25{ }^{\circ} \mathrm{C}$, respectively. This decrease of thickness was consistent with an increase of disorder upon heating. For the vesicle containing cholesterol, the value was $28 \AA$, which is markedly higher than for the above-mentioned system at the same temperature. It is noteworthy that myristic acid in the extended conformation (all-trans) would have a hydrophobic length of $13 \cdot \sin (109 / 2)$.
$1.54 \sim 16 \AA$; thus, for a bilayer that would be made of $\mathrm{My}$ in this conformation, the expected hydrophobic thickness would be twice, i.e., $32 \AA$.

These vesicles were further studied by using small-angle neutron scattering (SANS) allowing measuring the scattered intensity, $I(q)$, vs $q$, the scattering wave vector, as shown Figure 4. Data for the system containing 1-decanol at low temperature were not recorded but at $25^{\circ} \mathrm{C}$, one obtained a typical decrease of the intensity as a function of $q$ with a $q^{-2}$ power law as $q \rightarrow 0$ (Figure 4A). This was a typical behavior for vesicles which exhibited a diameter higher than $1 \mu \mathrm{m}$, in other words, a flat randomly oriented surface at the SANS scale length. One also observed a faint Bragg peak at around $q_{0}=0.03 \AA^{-1}$ suggesting that some vesicles were multilamellar (as mentioned above) with a spacing between bilayers, $d$, of the order of $210 \AA(d=$ $2 \pi / q_{0}$ ). The spectrum was similar for the vesicles containing cholesterol (Figure 4B) with a Bragg peak of smaller intensity at around $q_{0}=0.04 \AA^{-1}$ and, then, a spacing between bilayers of about $160 \AA$.

From these spectra, the Porod representation that returned $I(q) q^{4}$ as a function of $q$ was calculated as shown Figure $4 \mathrm{C}, \mathrm{D}$ for the vesicles containing 1-decanol and cholesterol at $25^{\circ} \mathrm{C}$, respectively. It was fitted with a model previously described ${ }^{17}$ with a unique parameter, the bilayer thickness. Note that this model and the fitting parameter also allowed plotting a curve on the above-mentioned spectra $I(q)$ vs $q$ and showed a good agreement with the experimental data except that Bragg peaks 
were not taken into account here. Data clearly showed a marked difference between both systems and the bilayer thickness determined was of 23 and $31 \AA$ for the vesicles containing 1-decanol and cholesterol, respectively. It is noteworthy that these values were $3 \AA$ higher than those calculated from the NMR data (see above).

CVC Measurements. We finally measured the critical vesicular concentration (CVC) in some of these vesicle-forming systems. The CVC represents the minimal concentration of fatty acids necessary to form vesicles, below which the sLCFA probably form micelles and/or are free as monomers. ${ }^{29}$ We employed the conductivity method since it is known that the break of conductivity upon increasing the surfactant concentration happens at the CVC. ${ }^{29}$ Data depicted in Figure 5 are commented hereafter. In the case of the $\mathrm{MyNaGu}$ vesicles at $\mathrm{pH} \mathrm{8}$, the break of conductivity occurred at around a concentration of My of $0.6 \mathrm{mM}$ (Figure 5A). This value is more than 100 times lower than in the case of decanoic acid vesicles, ${ }^{24}$ a fatty acid that has an alkyl chain shorter than that of myristic acid by four methylene groups. Upon addition of 1decanol in similar condition as described above (i.e., at $\mathrm{pH} 8$ ), the CVC decreased to $0.1 \mathrm{mM}$ (Figure 5B). The decrease of the CVC upon addition of alkanol has already been observed in the decanoic acid systems. ${ }^{24}$ Upon increasing the acyl chain length of the sLCFA, then in PaNaGu vesicles in the presence of 1-decanol the CVC again decreased to $0.07 \mathrm{mM}$. For vesicles produced with $\mathrm{PaNaGu}$ and 1-dodecanol, then increasing the alkanol chain length, no break of conductivity was observed; then the CVC could not be measured, indicating that it is probably lower than the detection limit of $10 \mu \mathrm{M}$. As expected because of higher hydrophobic interactions, the CVC were much lower in sLCFA vesicles than those observed for unsaturated or shorter chain fatty acids. ${ }^{24}$

\section{DISCUSSION}

Our present data allow showing that stable sLCFA vesicles can be obtained for a large range of composition and $\mathrm{pH}$ and are indeed formed by a fatty acid bilayer. These results are discussed hereafter and compared to those obtained for unsaturated and short chain fatty acids or phospholipid vesicles.

First of all, to our knowledge, this is the first time that stable vesicles are observed in sLCFA systems. This is allowed here since one used $\mathrm{GuHCl}$ that prevents the crystallization of sLCFA. $^{16,17}$ The use of this chaotropic agent should not be detrimental for applications such as for studying their foaming and emulsifying properties or in materials chemistry or even in biochemistry, if for instance, one wants studying the interactions of such vesicles with proteins. Indeed, proteins are known to be denatured in the presence of elevated amounts of $\mathrm{GuHCl}$. However, here, the concentration of $\mathrm{GuHCl}$ does not exceed that of the fatty acid, i.e., about few tens of $\mathrm{mM}$ in the $\mathrm{MyNaGu}$ system at $10 \mathrm{mg} / \mathrm{mL} \mathrm{My}$. This value is markedly lower than that used for denaturing proteins, about $6 \mathrm{M}$.

The present vesicles are then built using among the simplest and robust amphiphiles available. As in the case of short chain and unsaturated fatty acids, ${ }^{8}$ the formation of vesicles occurred upon decreasing the $\mathrm{pH}$ or adding an alkanol that acts as a cosurfactant. The formation mechanism of sLCFA vesicles is then probably very similar: it had been suggested that for short chain and unsaturated fatty acids, hydrogen bonds between the carboxylate and carboxylic groups coexisting at slightly basic $\mathrm{pH}$ or between the carboxylate and hydroxyl group of an alkanol would favor the bilayer structure (and then vesicles) versus that of micelles. In the present case using SLCFA, the carboxylate groups are stabilized by the guanidine moiety, preventing crystallization and decreasing the $\mathrm{pH}$ also allows the coexistence of carboxylate and carboxylic groups. In the same way, the hydroxyl group of 1-decanol can make hydrogen bonds with the sLCFA carboxylate group.

The sLCFA vesicles exhibit similar behavior than those made with phospholipids: they are formed by a sLCFA bilayer as evidenced by both solid state NMR and SANS. The bilayer thickness is comparable to that obtained for other lipids, being of the order of $2-3 \mathrm{~nm} .{ }^{28}$ It is noteworthy that the data determined from SANS are $3 \AA$ higher than those calculated from NMR. This difference can be attributed to the fact that SANS measures the whole bilayer thickness whereas NMR gives the hydrophobic bilayer thickness. To our knowledge, there is no any study reporting on the bilayer properties including the thickness as determined by physicochemical techniques in the case of short or unsaturated fatty acid chains.

The bilayers also exhibit a phase transition upon heating, sLCFA being embedded in a gel $\mathrm{L}_{\beta}$ state at low temperature with their acyl chains almost in an all-trans conformation and $\mathrm{L}_{\alpha}$ at higher temperature with disordered acyl chains. In the presence of cholesterol, the behavior is also similar as the one observed for phospholipid vesicles ${ }^{30}$ with a fluidificating/ rigidificating effect of the sterol. Here, it is noteworthy that the sterol is more easily incorporated within the sLCFA vesicles compared to the case of phospholipid vesicles without the need of any solvent, using sonication or multiple cycles of freezethawing. Indeed, no solvent was used to mix both sLCFA and cholesterol (sLCFA are very robust amphiphiles that are not submitted to chemical modifications such as hydrolysis as it is the case for phospholipids upon heating), and moreover, the fatty acid bilayer allows uploading up to $1 \mathrm{~mol}$ of sterol per sLCFA whereas a phospholipid bilayer can accommodate only $30 \mathrm{~mol} \%$ sterol. $^{30}$ Interestingly, mixtures of cholesterol and sodium soaps of palmitic acid have already been shown to form stable vesicles, ${ }^{31}$ and in that particular case, one can conclude that $\mathrm{GuHCl}$ is not necessary to get stable vesicles.

The CVC that are obtained in these sLCFA vesicles are of the order of those found in related systems with similar chain length and chemical structure, for instance, in lysophospholipid micellar dispersions. ${ }^{29,32,33}$ Also, the CVC obviously decreases when the acyl chain length of the sLCFA or that of the alkanol (when added) is increased. The CVC are then markedly lower than those obtained for short chain and unsaturated fatty acids. ${ }^{8,24}$ This may be of interest for applications in diluted systems.

Fatty acid vesicles are also of interest in the domain of protocells that are the first "cells" that appeared on the early Earth. ${ }^{8,22,34-36}$ It is well-known that several protocell models are based on fatty acid vesicles because these lipids were the most simple amphiphiles that could be present in prebiotic times. ${ }^{8}$ Although short chain or unsaturated fatty acids have long been put forward to play such a role, sLCFA could have been present on the early Earth. ${ }^{37,38}$ sLCFA vesicles could then also represent good protocell models of interest. The need of $\mathrm{GuHCl}$ is not detrimental for such systems since it is a very simple molecule that can have emerged from the oxidation of guanine and then be present in prebiotic times. The fact that sLCFA vesicles exhibit lower CVC than short chain or unsaturated fatty acids makes them of interest in a context of the appearance of life in large ponds of water in which dilution would have still afforded the formation of sLCFA vesicles 
whereas short chain or unsaturated fatty acids would not form vesicles but would remain as free monomers. Moreover, sLCFA vesicles resemble modern cells that are formed by phospholipids having acyl chain lengths of the order of $\mathrm{C}_{14}$ and $\mathrm{C}_{16}$ although some unsaturated chains may also be present. Finally, sLCFA vesicles made with cholesterol may be of interest for more evolved but still minimal cells, again exhibiting membrane properties similar to those of modern membranes.

In summary, we have demonstrated for the first time that sLCFA can form stable vesicles. This feature occurs in a broad range of composition and $\mathrm{pH}$. sLCFA vesicles exhibit properties similar as those observed for phospholipid vesicles; i.e., they are formed by a fatty acid bilayer of $2-3 \mathrm{~nm}$, the dynamics of their acyl chain depends on the temperature and the presence of cholesterol, and finally they exhibit relatively low CVC. Compared to existing systems forming vesicles, for instance phospholipids, sLCFA vesicles are simple and inexpensive, and with respect to short chain or unsaturated fatty acids, they exhibit lower CVC. Obviously, we can expect extending the formation of SLCFA vesicles to other systems in which these fatty acids no longer crystallize upon cooling, for instance, upon ion-pairing sLCFA with TBAOH or choline. Decreasing the $\mathrm{pH}$ and/or adding alkanols in these systems should yield vesicles as presently demonstrated, and we are planning investigating these systems in the future. Since sLCFA are green, cheap, and naturally available in large amounts, it is then believed that these simple vesicles could be of interest for various applications compared to vesicles made of phospholipids or short chain and unsaturated fatty acids.

\section{ASSOCIATED CONTENT}

\section{S Supporting Information}

The Supporting Information is available free of charge on the ACS Publications website at DOI: 10.1021/acs.langmuir.5b03627.

Additional phase diagrams (S.I.1) in the systems glycerol-1-myristate/MyNaGu and 1-tetradecanol/ $\mathrm{StNaGu}$ and simulated NMR spectra (S.I.2) in the systems 1-decanol/MyNaGu and cholesterol/MyNaGu at $25{ }^{\circ} \mathrm{C}(\mathrm{PDF})$

\section{AUTHOR INFORMATION}

\section{Corresponding Author}

*E-mail: jean-paul.douliez@bordeaux.inra.fr (J.-P.D.).

\section{Notes}

The authors declare no competing financial interest.

\section{ACKNOWLEDGMENTS}

Financial support from the TGIR-RMN-THC Fr3050 CNRS for conducting the research is gratefully acknowledged. We thank F. Cousin (LLB, CEA, France) for his help and stimulating discussions during the SANS measurements.

\section{REFERENCES}

(1) Du, J.; O'Reilly, R. K. Advances and challenges in smart and functional polymer vesicles. Soft Matter 2009, 5, 3544-3561.

(2) Bhattacharya, S.; Bajaj, A. Recent advances in lipid molecular design. Curr. Opin. Chem. Biol. 2005, 9, 647-655.

(3) Bhattacharya, S.; De, S. Vesicle Formation from Dimeric IonPaired Amphiphiles. Control over Vesicular Thermotropic and IonTransport Properties as a Function of Intra-amphiphilic Headgroup Separation. Langmuir 1999, 15, 3400-3410.
(4) Bhattacharya, S.; De, S.; Subramanian, M. Synthesis and Vesicle Formation from Hybrid Bolaphile/Amphiphile Ion-Pairs. Evidence of Membrane Property Modulation by Molecular Design. J. Org. Chem. 1998, 63, 7640-7651.

(5) Bhattacharya, S.; Haldar, S. Synthesis, Thermotropic Behavior, and Permeability Properties of Vesicular Membranes Composed of Cationic Mixed-Chain Surfactants. Langmuir 1995, 11, 4748-4757.

(6) Douliez, J.-P.; Gaillard, C. Self-assembly of fatty acids: from foams to protocell vesicles. New J. Chem. 2014, 38, 5142-5148.

(7) Golemanov, K.; Denkov, N. D.; Tcholakova, S.; Vethamuthu, M.; Lips, A. Surfactant Mixtures for Control of Bubble Surface Mobility in Foam Studies. Langmuir 2008, 24, 9956-9961.

(8) Morigaki, K.; Walde, P. Fatty acid vesicles. Curr. Opin. Colloid Interface Sci. 2007, 12 (2), 75-80.

(9) Cistola, D. P.; Atkinson, D.; Hamilton, J. A.; Small, D. M. Phase behavior and bilayer properties of fatty acids: hydrated 1:1 acid-soaps. Biochemistry 1986, 25, 2804-2812.

(10) Cistola, D. P.; Hamilton, J. A.; Jackson, D.; Small, D. M. Ionization and phase behavior of fatty acids in water: application of the Gibbs phase rule. Biochemistry 1988, 27, 1881-1888.

(11) Kralchevsky, P. A.; Danov, K. D.; Pishmanova, C. I.; Kralchevska, S. D.; Christov, N. C.; Ananthapadmanabhan, K. P.; Lips, A. Effect of the Precipitation of Neutral-Soap, Acid-Soap, and Alkanoic Acid Crystallites on the Bulk pH and Surface Tension of Soap Solutions. Langmuir 2007, 23, 3538-3553.

(12) Zana, R. Partial phase behavior and micellar properties of tetrabutylammonium salts of fatty acids: unusual solubility in water and formation of unexpectidly small micelles. Langmuir 2004, 20, $5666-5668$.

(13) Zana, R.; Schmidt, J.; Talmon, Y. Tetrabutylammonium Alkyl Carboxylate Surfactants in Aqueous Solution: Self-Association Behavior, Solution Nanostructure, and Comparison with Tetrabutylammonium Alkyl Sulfate Surfactants. Langmuir 2005, 21, 1162811636.

(14) Klein, R.; Kellermeier, M.; Drechsler, M.; Touraud, D.; Kunz, W. Solubilisation of stearic acid by the organic base choline hydroxide. Colloids Surf., A 2009, 338, 129-134.

(15) Klein, R.; Touraud, D.; Kunz, W. Choline carboxylate surfactants: biocompatible and highly soluble in water. Green Chem. 2008, 10, 433-435.

(16) Douliez, J.-P.; Houinsou-Houssou, B.; Fameau, A.-L.; Novales, B.; Gaillard, C. Self assembly of anastomosis-like superstructures in fatty acid/guanidine hydrochloride aqueous dispersions. J. Colloid Interface Sci. 2010, 341, 386-389.

(17) Fameau, A.-L.; Houinsou-Houssou, B.; Ventureira, J.; Novales, B.; Navailles, L.; Nallet, F.; Douliez, J.-P. Self assembly, foaming and emulsifying properties of sodium alkyl carboxylate/guanidine hydrochloride aqueous mixtures. Langmuir 2011, 27, 4505-4513.

(18) Blondeau, P.; Segura, M.; Perez-Fernandez, R.; de Mendoza, J. Molecular recognition of oxoanions based on guanidinium receptors. Chem. Soc. Rev. 2007, 36, 198-210.

(19) Pantos, A.; Tsogas, I.; Paleos, C. M. Guanidinium group: A versatile moiety inducing transport and multicompartmentalization in complementary membranes. Biochim. Biophys. Acta, Biomembr. 2008, $1778,811-823$.

(20) Davis, J. The description of membrane lipid conformation, order and dynamics by $2 \mathrm{H}-\mathrm{NMR}$. Biochim. Biophys. Acta, Rev. Biomembr. 1983, 737, 117-171.

(21) Grélard, A.; Guichard, P.; Bonnafous, P.; Marco, S.; Lambert, O.; Manin, C.; Ronzon, F.; Dufourc, E. J. Hepatitis B subvirus particles display both a fluid bilayer membrane and a strong resistance to freeze drying: a study by solid-state NMR, light scattering, and cryo-electron microscopy/tomography. FASEB J. 2013, 27, 4316-4326.

(22) Gebicki, J. M.; Hicks, M. Ufasomes are Stable Particles surrounded by Unsaturated Fatty Acid Membranes. Nature 1973, 243, 232-234.

(23) Hicks, M.; Gebicki, J. M. Microscopic studies of fatty acid vesicles. Chem. Phys. Lipids 1977, 20, 243-252. 
(24) Apel, C. L.; Deamer, D. W.; Mautner, M. N. Self-assembled vesicles of monocarboxylic acids and alcohols: conditions for stability and for the encapsulation of biopolymers. Biochim. Biophys. Acta, Biomembr. 2002, 1559, 1-9.

(25) Ouimet, J.; Croft, S.; Pare, C.; Katsaras, J.; Lafleur, M. Modulation of the Polymorphism of the Palmitic Acid/Cholesterol System by the $\mathrm{pH}$. Langmuir 2003, 19, 1089-1097.

(26) Pare, C.; Lafleur, M. Formation of Liquid Ordered Lamellar Phases in the Palmitic Acid/Cholesterol System. Langmuir 2001, 17, 5587-5594.

(27) Lafleur, M.; Fine, B.; Sternin, E.; Cullis, P.; Bloom, M. Smoothed orientational order profile of lipid bilayers by $2 \mathrm{H}$-nuclear magnetic resonance. Biophys. J. 1989, 56, 1037-1041.

(28) Douliez, J.; Leonard, A.; Dufourc, E. Restatement of order parameters in biomembranes: calculation of C-C bond order parameters from C-D quadrupolar splittings. Biophys. J. 1995, 68, 1727-1739.

(29) Israelachvili, J. Intermolecular and surface forces, 2nd ed.; Academic Press Ltd.: London, 1992.

(30) Vist, M. R.; Davis, J. H. Phase equilibria of cholesterol/ dipalmitoylphosphatidylcholine mixtures: $2 \mathrm{H}$ nuclear magnetic resonance and differential scanning calorimetry. Biochemistry 1990, 29, 451-464.

(31) Bastiat, G.; Oliger, P.; Karlsson, G. R.; Edwards, K.; Lafleur, M. Development of Non-Phospholipid Liposomes Containing a High Cholesterol Concentration. Langmuir 2007, 23, 7695-7699.

(32) Koynova, R.; Caffrey, M. An index of lipid phase diagrams. Chem. Phys. Lipids 2002, 115, 107-219.

(33) Koynova, R.; Caffrey, M. Phases and phase transitions of the phosphatidylcholines. Biochim. Biophys. Acta, Rev. Biomembr. 1998, 1376, 91-145.

(34) Monnard, P.-A.; Deamer, D. Nutrient Uptake by Protocells: A Liposome Model System. Origins Life Evol. Biospheres 2001, 31, 147155.

(35) Dzieciol, A. J.; Mann, S. Designs for life: protocell models in the laboratory. Chem. Soc. Rev. 2012, 41, 79-85.

(36) Mann, S. The Origins of Life: Old Problems, New Chemistries. Angew. Chem., Int. Ed. 2013, 52, 155-162.

(37) Rushdi, A. I.; Simoneit, B. R. T. Lipid Formation by Aqueous Fischer-Tropsch-Type Synthesis over a Temperature Range of 100 to $400{ }^{\circ} \mathrm{C}$. Origins Life Evol. Biospheres 2001, 31, 103-118.

(38) Simoneit, B. R. T. Prebiotic organic synthesis under hydrothermal conditions: an overview. Adv. Space Res. 2004, 33, 88-94. 